\title{
COMBINING ABILITY ESTIMATES FOR SOME AGRONOMIC, YIELD COMPONENTS AND BLAST REACTION TRAITS IN RICE (ORYZA SATIVA L.)
}

\author{
Anis, G.B.; H. M. Hassan; M. M. El-Malky and E. F. A. Arafat \\ Rice Research Section, Field Crops Research Inst., Agric. Research \\ Center, Egypt
}

\begin{abstract}
Combining ability analysis was carried out in rice, through A $6 \times 6$ diallel set analysis (excluding reciprocals) for some agronomic and yield components traits as well as blast reaction at the Experimental Farm of Sakha Agricultural Research station, Sakha, Kafr El-Sheikh, Egypt during 2015 and 2016 summer seasons. The ratio of variances due to general and specific combining abilities ( ó $^{2}$ GCA / ó $^{2}$ SCA) was found to be lower than unity for all studied characters, except 1000-grain weight, suggesting greater importance of non- additive genetic variance (over dominance or epitasis) in the inheritance of these characters. Obviously, highly significant and positive estimates of (GCA) effects were recorded for chlorophyll content in Large stigma rice variety, indicating that this entry could be used to improve such trait. Regarding to blast reaction, the rice varieties; Giza 177, Giza 179 and Giza 178 exhibited significant and highly significant negative values of GCA effects, while Large stigma and Baldo varieties showed the positive values of GCA effects. The results proved that the rice variety Large stigma considered as good combiner for improving the earliness, shortness and high chlorophyll content. Also, estimates of GCA effects showed that the parents, Giza 177, Giza 178 and Giza 179 were found to be good general combiners for blast reaction. Moreover, Giza 178 and Giza 179 were the best general combiners for grain yield/plant.

In addition, Blast reaction showed that five crosses were found to be high or highly significant and negative estimates of SCA, their estimated values were ranging from -0.70 for Giza $177 \times$ Large stigma to -0.83 for Giza $178 \times$ Large stigma. The crosses which gave negative significant values could be utilized in rice breeding program to improve this trait. Moreover, 5 crosses out of 15 combinations possessed significant desirable SCA effects for grain yield / plant involving two kinds of combinations between the parents of high $\mathrm{x}$ high GCA effects, such as SKC23819-192 x Baldo, suggesting that additive $x$ additive type of gene action play an important role in the inheritance of this trait.
\end{abstract}

From the foregoing results, the crosses Giza $177 \times$ Baldo, Giza $177 \times$ Large stigma, Giza $178 \times$ Large stigma and SKC23819-192 x Baldo could be utilized in rice breeding program to improve yield and its components and blast resistance traits. 
Key Words: rice, grain weight, blast reaction, grain yield, combining ability, heritability.

\section{INTRODUCTION}

Rice (Oryza sativa) is one of the important a cereal grain, and most widely consumed staple food for a large part of the world's human population (FAOSTAT, 2015). Also, is the most important grain with regard to human nutrition and caloric intake, providing more than one fifth of the calories consumed worldwide by humans (Smith, Bruce 1998). The grain yield in rice depending on different components, including 1000-grain weight which an important character to study not only because of its contribution to yield, but also because of its influence in rice marketing and trade (Gupta et al., 2006).

Grain size is usually evaluated by two criteria: grain weight and shape (Tan et al., 2000; Fan et al., 2006). Grain weight and grain shape (length, width, length/width ratio) are positively correlated characters (Fan et al., 2006). However, the economic way to get high yielding varieties it should have good grain shape, nutritional value, disease resistance, and stress tolerance Tan, et al., (2000). Study the inheritance and nature of relationships between grain length, grain shape and 1000-grain weight by (Mzengeza et al., 2010). Most of these ergonomically important traits are known to be genetically controlled by multiple genes Tan, et al., (2000).

Combining ability is defined as the ability of a parent line in hybrid combinations (Kambal and Webster, 1965). It plays an important role in selecting superior parents for hybrid combinations and in studying the nature of genetic variation (Duvick, 1999). It is a powerful method to measure the nature of gene action involved in quantitative traits (Baker, 1978). The authors defined GCA as the average performance of a line in hybrid combinations, while SCA as those instances in which certain hybrid combinations are either better or poorer than would be expected of the average performance of the parent inbred lines included. For random individuals, GCA is associated with additive effects of the genes, while SCA is related to dominance and epistatic effects (non-additive effects) of the genes (Sprague and Tatum, 1942). GCA effects represent the fixable component of genetic variance, and are important to develop superior genotypes. SCA represents a non-fixable component of genetic variation, it is important to provide information for hybrid performance. The GCA/SCA ratio is studied as parameter of the genetic variability in a diallel analysis. It estimates the type of gene action, which controls a particular characteristic (Quick, 1978; Sayed, 1978). When the ratio is high, it means the effect of the additive genes is prevalent. If the ratio is lower than one, it means the effect of nonadditive genes is prevalent in determining a particular character. If GCA variance is higher than 
SCA variance, the greater is the magnitude of additive genetic effects. Otherwise, the non-additive or dominant genetic variances are prevalent (Baker, 1978). The closer this ratio is to unity the greater the magnitude of additive genetic effects. Success of breeding [programs depends on the magnitude of genetic variability and the extent to which the advantageous characters. Therefore, the study of genetic variability in rice is not only essential for selecting variable genotypes and predicting that affect of selecting best genotypes but it will also aid breeders in simultaneous improvement of characters through selection (Patil, et al., 1993).

The combining ability analysis was done according to Griffing's (1956 b), Model 1, Method 2, where parents, and one set of F1's were included to estimate the effects of general (GCA) and specific (SCA) combining ability and variance components. The GCA: SCA ratio was estimated to study the performance of the effects and to measure the relative importance of additive gene or non-additive gene effects (Singh and Chaudhary, 1979). The objectives of this investigation, therefore, were to study the genetic variability for some agronomic, yield components and blast reaction traits.

\section{MATERIALS AND METHODS}

This study was conducted at Sakha Agricultural Research station, Sakha, Kafr El-Sheikh, Egypt during 2015 and 2016 growing seasons. Combining ability effects and genetic components were studied in six rice parents and their $\mathrm{F} 1_{\mathrm{s}}$ crosses, using six parents viz. Giza 177, Giza 178, Giza 179, SKC 23819-192, Baldo and Large Stigma (Table 1).

Table (1): parentage, origin and type of the six genotypes used in the experiment

\begin{tabular}{|l|c|c|c|}
\hline parents & Type & Parentage & Origin \\
\hline Giza 177 & Japonica & $\begin{array}{c}\text { (Giza 171/Yu mji } \\
\text { No.1//piNo.4) }\end{array}$ & Egypt \\
\hline Giza 178 & Indica-Japonica & Giza175/Milyang49 & Egypt \\
\hline Giza 179 & Indica-Japonica & $($ Gz1368/IRAT112) & Egypt \\
\hline SKC 23819-192 & Japonica & (M202/Sakha102) & Egypt \\
\hline Baldo & Japonica & Introduced line & Italy \\
\hline Large stigma & Indica-Japonica & Introduced line & China \\
\hline
\end{tabular}

The six parental varieties in this study were sown in the summer season of 2015 in three sowing dates, at 15 days intervals to overcome the difference of heading date among the parental varieties. After 30 days from sowing, seedlings of the parents were transplanted to the experimental field in three rows, of 5 meters long and $20 \times 20 \mathrm{~cm}$ apart between plants and rows. 
A half diallel cross was conducted among the six parents in 2015 growing season to produce $15 F_{1}$ cross. The six parental varieties and the resulting $15 \mathrm{~F}_{1}$ crosses were evaluated and arranged in a Randomized Complete Block Design (RCBD) experiment with three replications in 2016 growing season. Each replication contained 21 genotypes, one row per each genotypes, each row had 25 individual plants. Data were collected for following agronomic traits; days to maturing (days), plant height $(\mathrm{cm})$, number of panicles/plant, Chlorophyll content (SPAD), panicle length $(\mathrm{cm})$, number of filled grains/panicle, 1000-grain weight (gm), grain yield /plant (g) and blast reaction. Data were analyzed according to (Griffing analysis, 1956), method-2, model-1. This is a fixed model and was considered most appropriate as its all requirements were met by the experiment. Variances due to general and specific combining ability were estimated. All recommended cultural practices were followed to obtain normal growth of the crop according to RRTC (2015).

\section{RESULTS AND DISSECTION}

\section{Mean performance:}

Mean performance of studied characters for parents and their $F_{1}$ crosses are presented in Table (2). Regarding to number of days to heading, the rice variety Large Stigma and cross (Baldo $x$ Large stigma) showed the lowest values 81 and 88 days, respectively, while the rice genotypes; Giza 178, Giza 177 x Giza 178, Giza 177 x Giza 179, Giza 178 x Giza 179 and Giza 179 x Baldo recorded the highest values of number of days to heading. For plant height, Large stigma rice variety gave the shortest plant $86.3 \mathrm{~cm}$ while, Giza 178 and the hybrid combinations; SKC23819-192 x Baldo, Giza 177 x SKC23819-192 and Giza 177 x Baldo gave the tallest plants 105, 121.2, 117 and $115.2 \mathrm{~cm}$, respectively. Concerning chlorophyll content, the rice genotypes, Giza 178, large stigma, Baldo x Large stigma, SKC23819-192x Large stigma, SKC23819-192 x Baldo and Giza $179 \times$ Baldo showed the highest values of chlorophyll content in their flag leaf. On the other hand, Baldo and SKC23819-192 exhibited the lowest values.

The rice varieties Baldo and Large stigma were susceptible to blast disease, while the other rice genotypes including parents and their $F_{1}$ crosses were resistant to rice blast. The rice varieties; Giza 179, Giza 178 and crosses (Giza 177 x SKC23819-192) and (SKC23819-192 x Baldo) recorded the highest values of number of panicles plant/plant. While, Large stigma and Baldo showed the lowest values. For panicle length, Baldo and (Giza 178 x SKC23819-192) gave the longest panicle. On the other hand, (Giza $177 \times$ Giza 178) gave the shortest one. Regarding to number of filled grains 
panicle/plant, (Giza $177 \times$ Large stigma) and (Giza $177 \times$ Baldo) exhibited the highest values 198 and 191, while Large stigma recorded the lowest value 118. The genotypes; Baldo, SKC23819-192, Large stigma, (Baldo $x$ Large stigma) and (SKC23819-192 x Baldo) showed the highest values of 1000-grain weight 37.2, 35.4, 34.7, 35.7 and $33.6 \mathrm{~g}$, respectively. While, Giza 178 recorded the lowest value (21.2g).

Table (2): Mean performance of parents and their $F_{1}$ 's crosses for all studied traits during 2016 season

\begin{tabular}{|c|c|c|c|c|c|c|}
\hline Genotypes & $\begin{array}{c}\text { Days to } \\
\text { heading (day) }\end{array}$ & $\begin{array}{c}\text { Plant } \\
\text { height } \\
\text { (cm) }\end{array}$ & $\begin{array}{l}\text { Chlorophyll content } \\
\text { (SPAD) }\end{array}$ & $\begin{array}{c}\text { Number of } \\
\text { panicles/ } \\
\text { Plant }\end{array}$ & $\begin{array}{c}\text { Panicle } \\
\text { length }(\mathrm{cm})\end{array}$ & $\begin{array}{c}\text { Number of } \\
\text { Filled grains/ } \\
\text { panicle }\end{array}$ \\
\hline Giza 177 & 94 & 101.5 & 43.8 & 20 & 20.2 & 125 \\
\hline Giza 178 & 104 & 105.7 & 47.3 & 23 & 22.2 & 160 \\
\hline Giza 179 & 92 & 99 & 45.4 & 24 & 23.3 & 170 \\
\hline SKC 23819-192 & 99 & 102 & 38.2 & 21 & 23.3 & 161 \\
\hline Baldo & 98 & 97.2 & 36.4 & 17 & 24.3 & 138 \\
\hline Large stigma & 81 & 86.3 & 48.5 & 15 & 21.4 & 118 \\
\hline Giza 177 x Giza 178 & 103 & 96.3 & 42.6 & 24 & 17.8 & 123 \\
\hline Giza 177 X Giza 179 & 102 & 99.5 & 44.5 & 23 & 20.5 & 123 \\
\hline Giza 177 X SKC 23819-192 & 92 & 117 & 50.9 & 25 & 23.2 & 177 \\
\hline Giza 177 X Baldo & 98 & 115.2 & 48 & 24 & 23.4 & 191 \\
\hline Giza 177 X Large stigma & 102 & 1111.2 & 44.6 & 24 & 22.4 & 198 \\
\hline Giza 178 X Giza 179 & 101 & 98.8 & 42.8 & 23 & 21.3 & 141 \\
\hline Giza 178 X SKC 23819-192 & 96 & 105 & 44.5 & 25 & 26 & 112 \\
\hline Giza 178 X Baldo & 95 & 94.7 & 42.5 & 23 & 20.3 & 136 \\
\hline Giza $178 \mathrm{X}$ Large stigma & 100 & 95.6 & 43.4 & 22 & 21.4 & 146 \\
\hline Giza 179 X SKC 23819-192 & 96 & 113.9 & 45.8 & 22 & 22.8 & 130 \\
\hline Giza $179 \mathrm{X}$ Baldo & 101 & 101 & 52.4 & 22 & 22.4 & 179 \\
\hline Giza $179 \mathrm{X}$ Large stigma & 92 & 102.6 & 48.7 & 23 & 20.9 & 166 \\
\hline SKC 23819-192 x Baldo & 96 & 121.2 & 52.2 & 25 & 25.2 & 158 \\
\hline SKC 23819-192 x Large stigma & 92 & 103.2 & 52.7 & 23 & 22.3 & 111 \\
\hline Baldo $\mathrm{x}$ Large stigma & 88 & 96.4 & 53.7 & 24 & 23.3 & 135 \\
\hline L.S.D. at $0.05 \%$ & 1.49 & 1.31 & 1.34 & 1.57 & 0.77 & 5.97 \\
\hline $0.01 \%$ & 2 & 1.76 & 1.8 & 2.1 & 1.03 & 7.99 \\
\hline
\end{tabular}

Table (2): Continued

\begin{tabular}{|c|c|c|c|c|c|c|c|}
\hline Genotypes & $\begin{array}{l}\text { Number of } \\
\text { Filled grains/ } \\
\text { panicle }\end{array}$ & $\begin{array}{l}1000- \\
\text { grain } \\
\text { weight } \\
\text { (g) }\end{array}$ & $\begin{array}{c}\text { Grain } \\
\text { yield/ } \\
\text { plant } \\
\text { (g) }\end{array}$ & $\begin{array}{l}\text { Grain } \\
\text { length } \\
(\mathrm{mm})\end{array}$ & $\begin{array}{l}\text { Grain } \\
\text { width } \\
(\mathrm{mm})\end{array}$ & $\begin{array}{l}\text { Grain } \\
\text { shape } \\
(\mathrm{mm})\end{array}$ & $\begin{array}{c}\text { Blast } \\
\text { reaction }\end{array}$ \\
\hline Giza 177 & 125 & 28.1 & 36.4 & 7.6 & 3.3 & 2.3 & 2 \\
\hline Giza 178 & 160 & 21.2 & 45.4 & 7.6 & 3 & 2.5 & 2 \\
\hline Giza 179 & 170 & 27.5 & 45.4 & 7.9 & 3.2 & 2.8 & 2 \\
\hline SKC 23819-192 & 161 & 35.4 & 44.8 & 9.8 & 3.8 & 2.6 & 2 \\
\hline Baldo & 138 & 37.2 & 33.3 & 10 & 4 & 2.5 & 4 \\
\hline Large stigma & 118 & 34.7 & 30.5 & 9.5 & 3.5 & 2.7 & 4 \\
\hline Giza 177 x Giza 178 & 123 & 26.7 & 61.3 & 8.2 & 3.6 & 2.3 & 1 \\
\hline Giza 177 X Giza 179 & 123 & 28 & 51 & 8.5 & 3.8 & 2.2 & 1 \\
\hline Giza 177 X SKC 23819-192 & 177 & 31.1 & 54 & 7.8 & 3.8 & 2.1 & 2 \\
\hline Giza 177 X Baldo & 191 & 28 & 68 & 7.6 & 3.5 & 2.2 & 1 \\
\hline Giza 177 X Large stigma & 198 & 27.3 & 73.7 & 8 & 3.7 & 2.2 & 1 \\
\hline Giza 178 X Giza 179 & 141 & 27.8 & 51 & 8.4 & 3.7 & 2.2 & 2 \\
\hline Giza 178 X SKC 23819-192 & 112 & 25.3 & 76.7 & 8.4 & 3.8 & 2.2 & 2 \\
\hline Giza 178 X Baldo & 136 & 26.6 & 64.3 & 8 & 3.5 & 2.3 & 1 \\
\hline Giza 178 X Large stigma & 146 & 27.1 & 68 & 8.3 & 3.7 & 2.3 & 1 \\
\hline Giza 179 X SKC 23819-192 & 130 & 29 & 52.3 & 8.5 & 3.6 & 2.4 & 1 \\
\hline Giza 179 X Baldo & 179 & 28.3 & 65 & 7.9 & 3.6 & 2.3 & 2 \\
\hline Giza 179 X Large stigma & 166 & 28 & 51 & 7.9 & 3.5 & 2.3 & 1 \\
\hline SKC 23819-192 x Baldo & 158 & 33.6 & 74.7 & 8.3 & 3.5 & 2.4 & 1 \\
\hline SKC 23819-192 x Large stigma & 111 & 32.9 & 52.9 & 8.4 & 3.5 & 2.4 & 2 \\
\hline Baldo x Large stigma & 135 & 35.7 & 47.3 & 8.5 & 3.6 & 2.4 & 3 \\
\hline L.S.D. at $0.05 \%$ & 5.97 & 0.75 & 3.06 & 0.21 & 0.17 & 0.18 & 1.01 \\
\hline $0.01 \%$ & 7.99 & 1 & 4.09 & 0.28 & 0.23 & 0.24 & 1.34 \\
\hline
\end{tabular}

Concerning grain yield plant/plant, the genotypes Giza $178 \times$ SKC23819-192, SKC23819-192 x Baldo, and Giza 177 x Large stigma 
gave the highest values $76.7,74.7$ and $73.7 \mathrm{~g}$ plant/plant. On the other hand Large stigma and Baldo recorded the lowest values of grain yield plant/plant 30.5 and $33.3 \mathrm{~g}$, respectively. The results obtained are in agreement with that reported by Ramalingam et al. (1993).

The rice varieties; Giza 177, Giza 178 and Giza 179 recorded the lowest values for grain length and grain shape traits, while, SKC23819-192 and Baldo gave the highest values for grain width trait. In addition, the crosses (Giza 177 x SKC23819-192) and (Giza $177 \times$ Baldo) recorded the lowest values and desirable of grain length, while, (Giza 177 x Giza 179), (Giza 177 x SKC23819-192) and (Giza 178 x SKC23819-192) gave the highest values for grain width trait. On the other hand, (Giza 177 x SKC23819-192) and (Giza 177 x Baldo) gave the lowest values for grain shape trait.

\section{Analysis of Combining ability:}

Estimates of both general (GCA) and specific combining ability (SCA) variances for studied characters are presented in Tables (3).

Both general and specific combining ability variances were found to be highly significant for all characters, indicating the importance of both additive and non-additive genetic variance in determining the inheritance of the studied characters i.e. no. of days to heading, plant height, chlorophyll content, blast reaction, number of panicles plant/plant, panicle length, number of filled grains panicle/plant, 1000grain weight and grain yield plant/plant.

Table (3): Analysis of variance for all studied traits of six rice parents and $F_{1}$ crosses of rice

\begin{tabular}{|c|c|c|c|c|c|c|c|}
\hline S.O.V. & D.f & $\begin{array}{l}\text { Days to } \\
\text { heading } \\
\text { (day) }\end{array}$ & $\begin{array}{l}\text { Plant } \\
\text { height } \\
(\mathrm{cm})\end{array}$ & $\begin{array}{l}\text { Chlorophyll } \\
\text { content } \\
\text { (SPAD) }\end{array}$ & $\begin{array}{c}\text { Number } \\
\text { of } \\
\text { panicles/ } \\
\text { plant }\end{array}$ & $\begin{array}{l}\text { Panicle } \\
\text { length } \\
(\mathrm{cm})\end{array}$ & $\begin{array}{l}\text { Number of } \\
\text { Filled } \\
\text { grains/ } \\
\text { panicle }\end{array}$ \\
\hline Rep. & 2 & 0.08 & 2.08 & 1.74 & 0.14 & 0.24 & 0.49 \\
\hline Genotypes & 20 & $93.16^{* \star}$ & $217.39^{* *}$ & $64.75^{* *}$ & $19.25^{\star *}$ & $10.10^{\star *}$ & $2025.75^{\star \star}$ \\
\hline Parents & 5 & $191.63^{* *}$ & $133.99^{* *}$ & $72.48^{* *}$ & $37.25^{\star \star}$ & $6.60^{* *}$ & $1379.33^{* *}$ \\
\hline Crosses & 14 & $59.92^{* \star}$ & $227.88^{* *}$ & $51.61^{* *}$ & $3.23^{*}$ & $11.97^{* *}$ & $2393.65^{\star *}$ \\
\hline P. vs C $(\mathrm{H})$ & 1 & $66.05^{\star \star}$ & $487.52^{\star *}$ & $210.08^{* *}$ & $153.52^{* *}$ & $0.81^{*}$ & $107.30^{\star *}$ \\
\hline Error & 40 & 0.82 & 0.63 & 0.67 & 0.90 & 0.22 & 13.12 \\
\hline GCA & 5 & $58.37^{* *}$ & $107.34^{\star \star}$ & $11.96^{\star \star}$ & $5.93^{\star \star}$ & $6.36^{\star \star}$ & $243.88^{* *}$ \\
\hline SCA & 15 & $21.94^{\star \star}$ & $60.83^{\star \star}$ & $24.79^{* \star}$ & $6.57^{\star \star}$ & $2.35^{\star \star}$ & $819.04^{\star \star}$ \\
\hline Error term & 40 & 0.27 & 0.21 & 0.22 & 0.30 & 0.10 & 4.37 \\
\hline GCA/SCA ratio & --- & 0.33 & 0.22 & 0.06 & 0.11 & 0.34 & 0.04 \\
\hline
\end{tabular}

, ${ }^{* *}$ significant at 0.05 and 0.01 levels, respectively.

The relative importance of each variance was determined using GCA /SCA ratio of mean squares. The GCA /SCA ratio was found to be lower than unity for all studied characters except 1000-grain weight, suggesting greater importance of non- additive genetic variance (over dominance or epitasis) in the inheritance of these characters. The results obtained are in agreement with that reported by Ramalingam, 
et al. (1993), Bansal et al. (2000) and Sharam, Mani (2001) and ElMalky and Elamawi (2013) stated that the non-additive genetic variance is more important for the inheritance of number of days to heading and plant height. While GCA/SCA ratio was greater than unity for 1000-grain weight suggesting greater importance of additive genetic variance in the inheritance of this character, then, it could be concluded that selection procedures based on the accumulation of additive effect would be successful in improving these traits. The results obtained are in agreement with that reported by Hammoud (1996), EL-Refaee (2002), EL-Abd et al. (2003), El-Mowafi (2003) and Kolom et al. (2014).

Table (3): Continued

\begin{tabular}{|c|c|c|c|c|c|c|c|}
\hline S.O.V. & D.f. & $\begin{array}{c}\text { 1000-grain } \\
\text { weight } \\
\text { (g) }\end{array}$ & $\begin{array}{c}\text { Grain yield/ } \\
\text { plant } \\
\text { (g) }\end{array}$ & $\begin{array}{c}\text { Grain } \\
\text { length } \\
(\mathrm{mm})\end{array}$ & $\begin{array}{l}\text { Grain } \\
\text { width } \\
(\mathrm{mm})\end{array}$ & $\begin{array}{l}\text { Grain } \\
\text { shape } \\
(\mathrm{mm})\end{array}$ & $\begin{array}{c}\text { Blast } \\
\text { reaction }\end{array}$ \\
\hline Rep. & 2 & 0.33 & 2.84 & 0.01 & 0.00 & 0.01 & 0.21 \\
\hline Genotypes & 20 & $48.10^{* \star}$ & $538.52^{\star \star}$ & $1.38^{\star \star}$ & $0.16^{\star \star}$ & $0.11^{\star \star}$ & $1.95^{\star \star}$ \\
\hline Parents & 5 & $112.84^{\star \star}$ & $135.89^{\star \star}$ & $3.96^{\star \star}$ & $0.46^{\star \star}$ & $0.10^{\star \star}$ & $3.78^{\star \star}$ \\
\hline Crosses & 14 & $25.83^{\star}$ & $298.89^{\star \star}$ & $0.25^{\star}$ & $0.04^{\star}$ & $0.03^{*}$ & $0.89^{*}$ \\
\hline P.VsC (H) & 1 & $35.61^{\star \star}$ & $5906.41^{\star \star}$ & $4.16^{\star \star}$ & $0.29^{\star \star}$ & $1.10^{\star \star}$ & $7.55^{\star \star}$ \\
\hline Error & 40 & 0.21 & 3.43 & 0.02 & 0.01 & 0.01 & 0.37 \\
\hline GCA & 5 & $49.60^{\star \star}$ & $62.11^{\star \star}$ & $0.74^{\star \star}$ & $0.05^{\star}$ & $0.04^{\star}$ & $1.10^{\star \star}$ \\
\hline SCA & 15 & $4.83^{\star}$ & $218.63^{\star \star}$ & $0.36^{\star}$ & $0.06^{\star \star}$ & $0.03^{*}$ & $0.50^{\star}$ \\
\hline Error & 40 & 0.07 & 1.14 & 0.01 & 0.00 & 0.00 & 0.12 \\
\hline GCA/SCA ratio & --- & 1.30 & 0.03 & 0.26 & 0.12 & 0.16 & 0.32 \\
\hline
\end{tabular}

\section{General Combining Ability Effects (âi):}

Estimates of the general combining ability effects of the individual parental genotypes for vegetative traits are given in Tables (4). Highly significant and positive (ĝi) were observed for the chlorophyll content, number of panicles/plant, panicle length, number of filled grains/panicle, 1000-grain weight and grain yield/plant while, highly significant and negative values were recorded for no. of days to heading, plant height and blast reaction which are desirable for improvement of these traits in breeding programs since the low mean values are the target of breeder. The results obtained are in agreement with that reported by and El-Malky and Elamawi (2013), Quaqua et al. (2015) and Owere et al. (2016) also Gangopadhyay and Mohanty (1983) also reported the data on resistance to Pyricularia oryzae from a seven-parent diallel, including reciprocals, indicated that the resistant varieties were good general combiners in crosses with one moderately resistant and three susceptible varieties. With respect to no. of days to heading, the rice variety Large stigma showed highly significant and negative general combining ability effects, indicating that this parent could be considered as good combiner for the improvement of this trait. Regarding plant height, the rice genotypes, Large stigma, Giza 178 and Giza 179 showed highly significant and negative general 
combining ability effects with values $-4.93,-2.42$ and -0.90 respectively. The negative values of (⿳⺈⿴囗十) for this trait is required from breeding point of view since it refers to the short stature plant type. For chlorophyll content, highly significant and positive estimates of (ĝi) were recorded for Large stigma, indicating that this parent could be used to improve such trait.

Table (4): Estimates of general and specific combining ability effects of parents and their $\mathrm{F} 1$ crosses for all studied traits

\begin{tabular}{|c|c|c|c|c|c|c|}
\hline Parents and crosses & $\begin{array}{c}\text { Days to } \\
\text { heading } \\
\text { (day) }\end{array}$ & $\begin{array}{l}\text { Plant } \\
\text { height } \\
(\mathrm{cm})\end{array}$ & $\begin{array}{c}\text { Chloroph } \\
\text { yll } \\
\text { content } \\
\text { (SPAD) }\end{array}$ & $\begin{array}{c}\text { Number } \\
\text { of } \\
\text { panicles/p } \\
\text { lant }\end{array}$ & $\begin{array}{l}\text { Panicle } \\
\text { length } \\
\text { (cm) }\end{array}$ & $\begin{array}{c}\text { Number } \\
\text { of Filled } \\
\text { grains/pa } \\
\text { nicle }\end{array}$ \\
\hline \multicolumn{7}{|l|}{ GCA } \\
\hline Giza 177 & $1.24^{\star \star}$ & $2.63^{\star \star}$ & $-0.60^{\star \star}$ & 0.29 & $-1.02^{\star \star}$ & $3.61^{\star \star}$ \\
\hline Giza 178 & $3.52^{\star \star}$ & $-2.42^{\star \star}$ & $-1.57^{\star \star}$ & $0.75^{\star \star}$ & $-0.60^{\star \star}$ & $-6.85^{\star \star}$ \\
\hline Giza 179 & 0.23 & $-0.90^{\star \star}$ & $0.27^{\star}$ & 0.50 & $-0.20^{\star}$ & $5.74^{\star \star}$ \\
\hline SKC 23819-192 & -0.36 & $5.40^{\star \star}$ & -0.07 & $0.54^{\star}$ & $1.26^{\star \star}$ & $-2.81^{\star \star}$ \\
\hline Baldo & 0.10 & 0.23 & -0.17 & $-0.62^{\star \star}$ & $0.91^{\star \star}$ & $5.24^{\star \star}$ \\
\hline Large stigma & $-4.73^{\star \star}$ & $-4.93^{\star \star}$ & $2.13^{\star \star}$ & $-1.46^{\star \star}$ & $-0.36^{\star \star}$ & $-4.93^{\star \star}$ \\
\hline $\begin{array}{r}\text { L.S.D. at } 0.05 \% \\
\text { at } 0.01 \%\end{array}$ & $\begin{array}{l}0.58 \\
0.41\end{array}$ & $\begin{array}{l}0.25 \\
0.36\end{array}$ & $\begin{array}{l}0.26 \\
0.37\end{array}$ & $\begin{array}{l}0.51 \\
0.75\end{array}$ & $\begin{array}{l}0.14 \\
0.21\end{array}$ & $\begin{array}{l}1.13 \\
1.63\end{array}$ \\
\hline \multicolumn{7}{|l|}{ SCA } \\
\hline Giza 177 x Giza 178 & $1.65^{\star \star}$ & $-6.95^{\star \star}$ & $-1.38^{\star \star}$ & 0.10 & $-2.83^{\star \star}$ & $-21.49^{\star \star}$ \\
\hline Giza 177 X Giza 179 & $4.27^{\star \star}$ & $-5.27^{\star \star}$ & $-1.28^{\star \star}$ & 0.02 & $-0.56^{\star}$ & $-34.08^{\star \star}$ \\
\hline Giza 177 X SKC 23819-192 & $-5.14^{\star \star}$ & $5.97^{\star \star}$ & $5.45^{\star \star}$ & $1.64^{\star \star}$ & $0.69^{\star \star}$ & $28.46^{\star \star}$ \\
\hline Giza 177 X Baldo & 0.73 & $9.31^{\star \star}$ & $2.62^{\star \star}$ & $2.14^{\star \star}$ & $1.23^{\star \star}$ & $34.42^{\star \star}$ \\
\hline Giza 177 X Large stigma & $8.90^{\star \star}$ & $10.47^{\star \star}$ & $-3.05^{\star \star}$ & $2.31^{\star \star}$ & $1.47^{\star \star}$ & $51.92^{\star \star}$ \\
\hline Giza 178 X Giza 179 & 0.66 & $-0.86^{\star}$ & $-2.01^{\star \star}$ & $-0.77^{\star \star}$ & -0.22 & $-5.62^{\star \star}$ \\
\hline Giza 178 X SKC 23819-192 & $-3.43^{\star \star}$ & $-0.99^{\star \star}$ & $-0.01^{\star}$ & $1.18^{\star \star}$ & $3.03^{\star \star}$ & $-26.08^{\star \star}$ \\
\hline Giza 178 X Baldo & $-5.22^{\star \star}$ & $-6.12^{\star \star}$ & $-1.91^{\star \star}$ & $0.68^{\star \star}$ & $-2.29^{\star \star}$ & $-9.79^{\star \star}$ \\
\hline Giza $178 \times$ Large stigma & $4.95^{\star \star}$ & -0.06 & $-3.31^{\star \star}$ & 0.18 & 0.08 & $10.38^{\star \star}$ \\
\hline Giza 179 X SKC 23819-192 & 0.20 & $6.42^{\star \star}$ & $-0.51^{\star}$ & $-1.57^{\star \star}$ & $-0.57^{\star}$ & $-20.66^{\star \star}$ \\
\hline Giza 179 X Baldo & $4.41^{\star \star}$ & $-1.33^{\star \star}$ & $6.19^{\star \star}$ & $-0.40^{\star}$ & $-0.63^{\star \star}$ & $20.30^{\star \star}$ \\
\hline Giza 179 X Large stigma & 0.57 & $5.46^{\star \star}$ & $0.16^{\star}$ & $1.77^{\star \star}$ & $-0.79^{\star \star}$ & $17.80^{\star \star}$ \\
\hline SKC 23819-192 x Baldo & -0.01 & $12.53^{\star \star}$ & $6.26^{\star \star}$ & $2.56^{\star \star}$ & $0.75^{\star \star}$ & $7.84^{\star \star}$ \\
\hline SKC 23819-192 x Large stigma & $0.82^{\star}$ & -0.31 & $4.49^{\star \star}$ & $1.39^{\star \star}$ & $-0.91^{\star \star}$ & $-28.66^{\star \star}$ \\
\hline Baldo $\mathrm{x}$ Large stigma & $-3.64^{\star \star}$ & $-1.87^{\star \star}$ & $5.59^{\star \star}$ & $3.56^{\star \star}$ & $0.47^{\star}$ & $-12.37^{\star \star}$ \\
\hline $\begin{array}{l}\text { L.S.D. at } 0.05 \% \\
\text { at } 0.01 \%\end{array}$ & 0.78 & 0.68 & 0.70 & 0.29 & 0.40 & 3.12 \\
\hline
\end{tabular}

${ }^{*},{ }^{* \star}$ significant at 0.05 and 0.01 levels, respectively.

With respect to blast reaction the rice varieties; Giza 177, Giza 179 and Giza 178 exhibited significant and highly significant negative values of ( $\hat{g} \mathbf{i})$, while Large stigma and Baldo showed the positive values of (ĝi). From the above mentioned results, it could be concluded that the rice variety Large stigma considered as good combiner for improving the number of days to heading (earliness), plant height and chlorophyll content. Obviously, estimates of (ĝi) showed that the parents, Giza 177, Giza 178 and Giza 179 were found to be good general combiners for blast reaction (Table 4). 
Table (4): Continued

\begin{tabular}{|c|c|c|c|c|c|c|}
\hline Parents and crosses & $\begin{array}{c}1000- \\
\text { grain } \\
\text { weight } \\
(\mathrm{g})\end{array}$ & $\begin{array}{c}\text { Grain } \\
\text { yield/plant } \\
(\mathrm{g})\end{array}$ & $\begin{array}{l}\text { Grain } \\
\text { length } \\
(\mathrm{mm})\end{array}$ & $\begin{array}{l}\text { Grain } \\
\text { width } \\
(\mathrm{mm})\end{array}$ & $\begin{array}{l}\text { Grain } \\
\text { shape } \\
(\mathrm{mm})\end{array}$ & $\begin{array}{l}\text { Blast } \\
\text { reaction }\end{array}$ \\
\hline \multicolumn{7}{|l|}{ GCA } \\
\hline Giza 177 & $-1.15^{\star \star}$ & -0.19 & $-0.38^{\star \star}$ & 0.00 & $-0.13^{\star \star}$ & $-0.32^{\star \star}$ \\
\hline Giza 178 & $-3.82^{\star \star}$ & $3.72^{\star \star}$ & $-0.25^{\star \star}$ & $-0.11^{\star \star}$ & -0.01 & $-0.19^{\star}$ \\
\hline Giza 179 & $-1.30^{\star \star}$ & $2.23^{\star \star}$ & $-0.17^{\star \star}$ & $-0.07^{\star \star}$ & $0.06^{\star \star}$ & $-0.28^{\star \star}$ \\
\hline SKC 23819-192 & $2.02^{\star \star}$ & $-2.65^{\star \star}$ & $0.35^{\star \star}$ & $0.10^{\star \star}$ & 0.01 & -0.15 \\
\hline Baldo & $2.51^{\star \star}$ & 0.45 & $0.24^{\star \star}$ & $0.08^{\star \star}$ & 0.00 & $0.43^{\star \star}$ \\
\hline Large stigma & $1.73^{\star \star}$ & $-3.56^{\star \star}$ & $0.22^{\star \star}$ & 0.00 & $0.07^{\star \star}$ & $0.51^{\star \star}$ \\
\hline $\begin{array}{r}\text { L.S.D. at } 0.05 \% \\
\text { at } 0.01 \%\end{array}$ & $\begin{array}{l}0.14 \\
0.20 \\
\end{array}$ & $\begin{array}{l}0.58 \\
0.83 \\
\end{array}$ & $\begin{array}{l}0.04 \\
0.06\end{array}$ & $\begin{array}{l}0.03 \\
0.05\end{array}$ & $\begin{array}{l}0.03 \\
0.04\end{array}$ & $\begin{array}{l}0.19 \\
0.27\end{array}$ \\
\hline \multicolumn{7}{|l|}{ SCA } \\
\hline Giza 177 x Giza 178 & $2.17^{\star \star}$ & $3.18^{\star \star}$ & $0.47^{\star \star}$ & $0.13^{\star}$ & 0.09 & 0.01 \\
\hline Giza 177 X Giza 179 & $0.95^{\star \star}$ & -0.78 & $0.68^{\star \star}$ & $0.26^{\star \star}$ & -0.05 & 0.09 \\
\hline Giza 177 X SKC 23819-192 & $0.72^{\star \star}$ & $-2.66^{\star \star}$ & $-0.43^{\star \star}$ & $0.12^{\star}$ & $-0.17^{\star \star}$ & 0.30 \\
\hline Giza 177 X Baldo & $-2.87^{\star \star}$ & $13.12^{\star \star}$ & $-0.63^{\star \star}$ & $-0.19^{\star \star}$ & -0.06 & $-0.62^{\star}$ \\
\hline Giza 177 X Large stigma & $-2.75^{\star \star}$ & $22.80^{\star \star}$ & $-0.21^{\star \star}$ & $0.16^{\star \star}$ & $-0.13^{\star}$ & $-0.70^{\star}$ \\
\hline Giza 178 X Giza 179 & $3.42^{\star \star}$ & $-4.69^{\star \star}$ & $0.45^{\star \star}$ & $0.34^{\star *}$ & $-0.16^{\star \star}$ & 0.30 \\
\hline Giza 178 X SKC 23819-192 & $-2.43^{\star \star}$ & $16.09^{\star \star}$ & -0.06 & $0.20^{\star \star}$ & $-0.15^{\star \star}$ & $0.84^{\star \star}$ \\
\hline Giza 178 X Baldo & $-1.56^{\star \star}$ & $5.54^{\star \star}$ & $-0.36^{\star \star}$ & -0.08 & -0.04 & $-0.74^{\star}$ \\
\hline Giza 178 X Large stigma & -0.28 & $13.22^{\star \star}$ & 0.03 & $0.20^{* \star}$ & $-0.14^{\star \star}$ & $-0.83^{\star \star}$ \\
\hline Giza 179 X SKC 23819-192 & $-1.22^{\star \star}$ & $-1.87^{*}$ & -0.01 & -0.01 & -0.06 & -0.08 \\
\hline Giza 179 X Baldo & $-2.45^{\star \star}$ & $12.58^{\star \star}$ & $-0.48^{\star \star}$ & -0.01 & $-0.14^{\star \star}$ & -0.33 \\
\hline Giza 179 X Large stigma & $-1.90^{\star \star}$ & $2.59^{\star \star}$ & $-0.52^{\star \star}$ & -0.04 & $-0.18^{\star \star}$ & $-0.74^{\star}$ \\
\hline SKC 23819-192 x Baldo & $-0.47^{\star}$ & $17.36^{\star \star}$ & $-0.63^{\star \star}$ & $-0.25^{\star \star}$ & 0.00 & $-0.79^{\star \star}$ \\
\hline SKC 23819-192 x Large stigma & $-0.39^{*}$ & -0.42 & $-0.47^{\star \star}$ & $-0.17^{\star \star \star}$ & 0.00 & $-0.54^{*}$ \\
\hline Baldo $\mathrm{x}$ Large stigma & $1.92^{\star \star}$ & $-4.21^{\star \star}$ & $-0.27^{\star \star}$ & -0.01 & -0.05 & $0.55^{\star}$ \\
\hline $\begin{array}{r}\text { L.S.D. at } 0.05 \% \\
\text { at } 0.01 \%\end{array}$ & $\begin{array}{l}0.39 \\
0.56 \\
\end{array}$ & $\begin{array}{l}1.59 \\
2.29\end{array}$ & $\begin{array}{l}0.11 \\
0.16\end{array}$ & $\begin{array}{l}0.09 \\
0.13\end{array}$ & $\begin{array}{l}0.10 \\
0.13\end{array}$ & $\begin{array}{l}0.52 \\
0.75\end{array}$ \\
\hline
\end{tabular}

${ }^{*},{ }^{* *}$ significant at 0.05 and 0.01 levels, respectively.

Moreover, Giza 178 and Giza 179 were the best general combiners for grain yield/plant. High (ĝi) of Giza 178 for grain yield

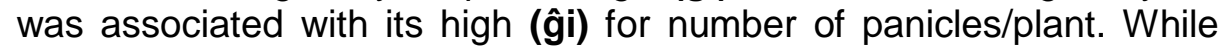
the high estimates of general combining ability of cultivar, Giza 179 for grain yield/plant was due to its highest estimates of number of panicles/plant and number of filled grains/panicle. The results also revealed that among the studied parents, Giza 177 followed by Giza 178 and Giza 179 were the best general combiners for short grain. Moreover, SKC 23819-192 and Baldo were the best general combiners for width grain. In addition, highly significant and positive estimates of general combining ability of grain shape were recorded for Giza 179 and Large stigma, indicating that these two parents were the greatest combiners for improving this trait. The results obtained are in agreement with that reported by Hammoud (1996), EL-Abd et al. (2003), El-Mowafi (2003) and Kolom et al. (2014)

\section{Specific Combining Ability Effects (ôii):}

Estimates of specific combining ability effects are shown in Table (4). In case of days to heading, six crosses showed significant and highly significant positive estimates of ( $\hat{g} \mathrm{ij})$ ranged between 0.82 for SKC23819-192 x Large stigma and 8.90 for Giza 177 x Large stigma. While, four crosses gave highly significant and negative (ĝij) 
with values varied from -3.43 for Giza $178 \times$ SKC23819-192 to -5.22 for Giza 178 x Baldo. The crosses which gave highly significant and negative estimates could be used as a donor to improve this trait. For plant height seven rice cross combinations showed significant and highly significant negative values of (解), indicating that these crosses may be useful in exploitation of heterosis due to their desirable plant stature.

Concerning chlorophyll content, Table (4), five crosses gave highly significant and positive of (ĝij). The best crosses were (Giza 177 x SKC23819-192), (Giza 179 x Baldo), (SKC23819-192 x Baldo), (SKC23819-192 $\times$ Large stigma) and (Baldo $\times$ Large stigma) their estimated values were $5.45,6.19,6.26,4.49$ and 5.59 , respectively. The combinations which showed significant and positive (ĝij) may be useful in exploitation of heterosis due to their desirable increase chlorophyll content. Blast reaction showed that (ĝij) of five crosses were high and highly significant negative ranging from -0.70 for (Giza $177 \times$ Large stigma) to -0.83 for (Giza $178 \times$ Large stigma). The crosses which gave negative significant values could be utilized in rice breeding program to improve this trait. The results obtained are in agreement with that reported by and El-Malky and Elamawi (2013), Quaqua et al. (2015) and Owere et al. (2016) also Gangopadhyay and Mohanty (1983) also reported the data on resistance to Pyricularia oryzae from a seven-parent diallel, including reciprocals, indicated that three specific cross combinations with good combining ability were identified. Additive genetic variance was more important than nonadditive.

As shown in Table (4), 5 crosses out of 15 combinations possessed significant desirable (ĝij) for grain yield / plant involving two kind of combinations between the parents of high and high (ĝi), such as (SKC23819-192 $\times$ Baldo) recorded high $\times$ high parental (ĝi), suggesting that additive $x$ additive type gene action. Manual and Palanisamy (1989) also reported interaction between positive alleles in crosses involving high $x$ high combiners which can be fixed in subsequent generations if no repulsion phase linkages are involved. Crosses like Giza 177 x Baldo, Giza 177 x Large stigma and Giza 178 $x$ SKC23819-192 showed high $x$ low or low $x$ high parental (ĝi), indicating the involvement of additive $x$ dominance or dominance $x$ additive genetic interaction. Peng and Virmani (1990) also reported about the possibility of interaction between positive alleles from good combiners and negative alleles from poor combiners in high $x$ low crosses and suggested exploitation of heterosis in $\mathrm{F} 1$ generation. Their high yield potential would be unfixable in succeeding generations. Similar results were also obtained by Dubey (1975). The cross Giza 
$178 \times$ Large stigma recorded low $\times$ low parental (ĝi) indicating over dominance and epistatic interactions.

In the present study, the crosses (Giza $177 \times$ Baldo) and (SKC23819-192 x Baldo) exhibited the highest (ĝij) for grain length. Similarly, (Giza 177 x Giza 179) and (Giza 178 x Giza 179) showed highest ( $\hat{\mathbf{g}} \mathbf{i j})$ for grain width. The crosses which gave negative for grain length and positive for grain width could be utilized in rice breeding program to improve this trait. The result is in agreement with the work of Hammoud (1996) and EL-Abd et al. (2003).

From the foregoing results, the crosses Giza $177 \times$ Baldo, Giza 177 x Large stigma, Giza 178 x Large stigma and SKC23819-192 x Baldo could be utilized in rice breeding program to improve yield and its components and blast resistance traits.

\section{REFERENCE}

Baker, R. J. 1978. Issues in diallel analysis. Crop Sci. 18:533-536.

Bansal, U.K.; R.G. Saini and N.S. Rani (2000). Heterosis and combining ability for yield, its components and quality traits in some scented rice (Oryza sativa L.). Tropical Agric., 77: 180 - 187.

Dubey, R.S. 1975. Combining ability in cigar filter tobacco. Indian J Genet. 35: 76 - 92.

Duvick, D. N. 1999. Commercial strategies for exploitation of heterosis. In: Coors J.G. \& Pandey, S. (eds.). The genetics and exploitation of heterosis in crops. ASA, CSS, and SSSA. Madison, Wisconsin, USA, pp. 19 - 29.

EL-Abd, A.B.; A.A. Abdallah and A.A. EL-Hissewy (2003). Studies on combining ability and herterosis for some physiological characters in rice. (Oryza sativa L.). The tenth Conference of Agronomy October, 7-10. Suez Canal University EL-Irish.

EL-Mowafi, H.F. and A.A. Abou Shousha (2003). Combining ability and heterosis analysis of diverse CMS lines in hybrid rice. J. Agric. Res. Tanta Univ., March 29(1):106-127.

El-Refaee, Y.Z.A. (2002). Genetical and Biochemical studies on heterosis and combining ability in rice. M.Sc. Thesis, Genetics Department, Fac. Of Agric., Kafr El-sheikh, Tanta University, Egypt.

Fan C, Zing Y, Mao H, Lu T, Han B, Xu C, Li X and Zhang Q. (2006). GS3, a major QTL for grain length and weight and minor QTL for grain width and thickness in rice, encodes a punitative transmembrane protein.Theoretical and Applied Genetics 112: 1164 -1171.

Gangopadhyay, S. and Mohanty, C. R. (1983). Combining ability for resistance to blast disease in rice. Current Science. 52 (20): 984 985.

Griffing, B. 1956 (b). Concept of general and specific combining ability in relation to diallel crossing systems. Aust. J. Biol. Sci., 9: 463 - 493. 
Griffing, J. B. (1956). Concept of general and specific combining ability in relation to diallel crossing system. Aust. J. Biol. Sci. 9: 43.

Gupta PK, Rustgi S and Kumar N. (2006). Genetic and molecular basis of grain size and grain number and its relevance to grain productivity in higher plants. Genome 49: 565 - 571.

Hammoud, S.A.A. (1996). Breeding studies on some rice characters. M.Sc. Thesis, Fac. Of Agric., Minufiya University Shibin El-Kom, Egypt.

Kambal, A. E. and O. J. Webster. 1965. Estimates of general and special combining ability in grain sorghum, Sorghum vulgare pers.Crop Sci. 5:521 - 523.

Kolom, R., S. Changkija and M. B. Sharma (2014). Combining ability analysis for yield and yield components in some important upland rice germplasme of Nagaland. Indian Journal of Hill Farming. 27(1): $118-125$.

Manuel, W.W. and Palanisamy, S. 1989. Line $x$ tester analysis of combining ability in rice. Oryza, 26(1):27-32.

Mzengeza T.R., P. Tongoona, J. Derera and A.S. Kumwenda (2010). Genetic analysis of grain size in F2 populations of crosses between Malawi rice landraces and NERICA varieties. Second Africa Rice Congress, Bamako, Mali.

Owere, L.; P. Tongoona; J. Derera and N. Wanyera. (2016). Combining Ability Analysis of Blast Disease Resistance and Agronomic Traits in Finger Millet [Eleusine coracana (L.) Gaertn]. Journal of Agricultural Science 8(11): 138 - 146.

Patil, P.A.; C.R. Mahajan, S.S. Mehetre and D.N. Hajare (1993). Analysis of variability and heritability in upland rice. Oryza., 30, 154 - 156

Peng, J.Y. and Virmani, S.S. 1990. Combining ability for yield and four related traits in relation to breeding in rice. Oryza, 27(1):1-10.

Quaqua S. M.; Hussein, A. S. and M. D. Laing (2015). Combining ability and gene action of three components of horizontal resistance against rice blast. Euphytica 206: 805 - 814 .

Quick, J. S. 1978. Combining ability and interrelationship among an international array of durum wheat. In: S. Ramanujam (ed.). Proceedings of the Fifth International Wheat Genetic Symposium. New Delhi, pp. 634 - 647.

Ramalingam, J. P. Vivekanandan and C.Vanniarajan (1993). Combining ability analysis in low land early rice. Crop. Research, 6(2): 228 233.

RRTC, 2015. Proceeding Rice Research and Training Center, Sakha, Kafr El-Sheikh, Egypt.

Sayed, H. I. 1978. Combining ability for yield and its component characters in wheat. In: S. Ramanujam (ed.). Proceeding of the Fifth International Wheat Genetic Symposium. New Delhi, pp. 634 636.

Sharma, R. K. and S. C. Mani (2001). Combining ability studies for grain yield and other associated characters in basmati rice (Oryza sativa L.). Crop improvement, 28(2): 236 - 243. 
Singh, R. K. and B. D. Chaudhary. 1979. Biometrical Methods in Quantitative Genetic Analysis. Kalyani Publ., New Delhi.

Smith, Bruce D. (1998). The Emergence of Agriculture. Scientific American Library, A Division of HPHLP, New York, ISBN 0-71676030-4.

Sprague, G. F. and L. A. Tatum. 1942. General vs. specific combining ability in single crosses of corn. J. Amer. Soc. Agron. 34: 923 932.

Tan Y.F., Xing Y.Z., Li J.X., Yu S.B., Xu C.G. and Zhang Q. (2000). Genetic bases of appearance quality of rice grains in Shanyou 63, an elite rice hybrid. Theoretical and Applied Genetics 101: 823 829.

Tan, Y.F. et al. (2000). Genetic bases of appearance quality of rice grains in Shanyou 63, an elite rice hybrid. Theor. Appl. Genet. 101, 823 829.

Yang, L.S. et al. (2001) Research progress of rice grain type and its inheritance. J. Anfui Agric. Sci. 29, 164 - 167.

تقديرات القدرة على التألف لبعض الصفات الخضرية والمحصولية والمقاومة

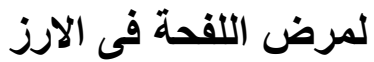

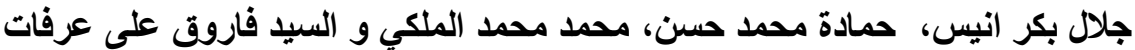

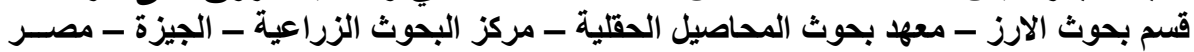

$$
\text { الملخـص العـربى }
$$

تمت در اسة القدرة على التآلف لصفات وزن الحبوب و المقاومة لمرض اللفحة و الصفات

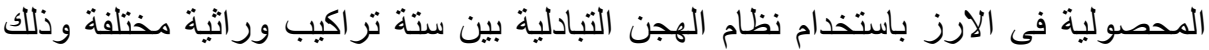

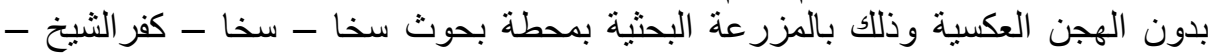

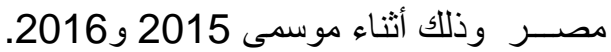

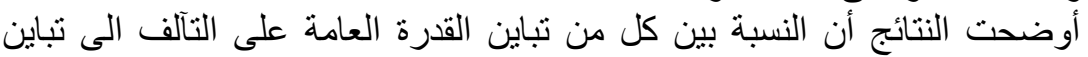

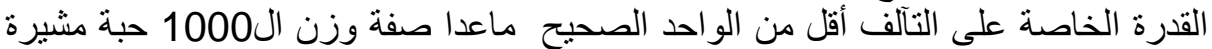

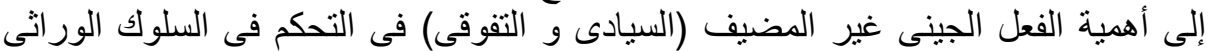

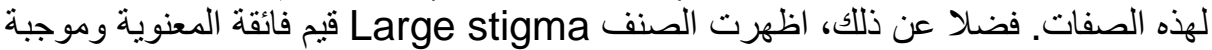

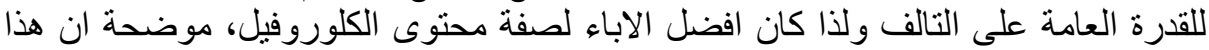

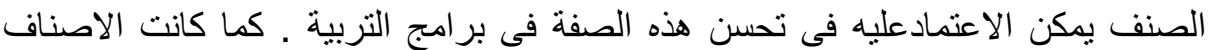

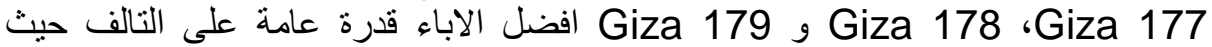

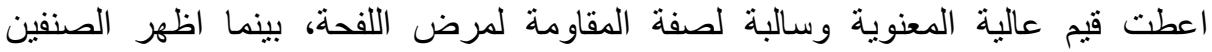
Baldo المقاومة لمرض اللفحة. ومن جانب اخر، وجد ان الصنف Large stigma افضل التضل الاباء قدرة عامة على التالف لصفات التبكير، قصر النبات و وحنو محن الكلوروفيل و عليه يمكن 
Giza و Giza استخدامه في برامج التربية لتحسن هذه الصفات.كما كانت الاصناف 178

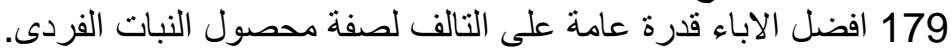

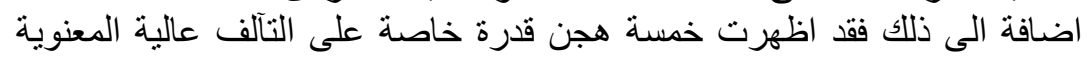

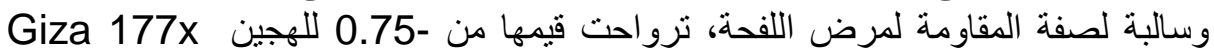
Large stigma

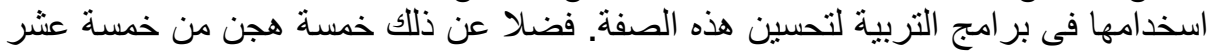

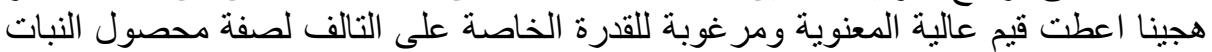

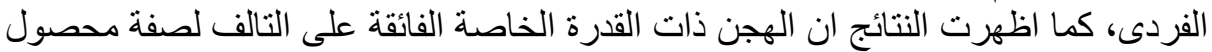

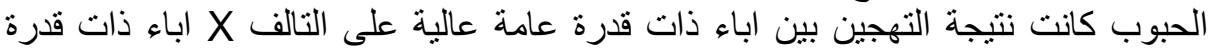

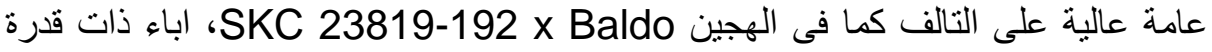

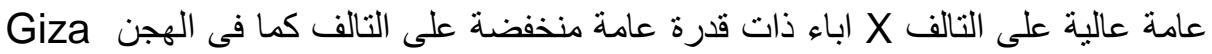
Giza 178 x SKC 23818- و Giza 177 x Large stigma،177 x Baldo

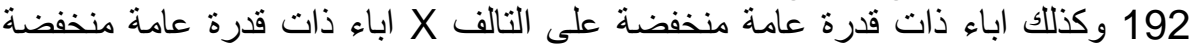

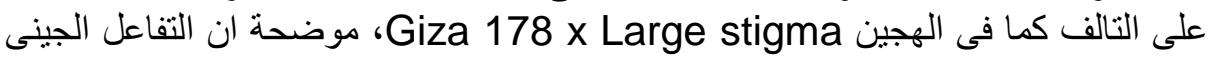

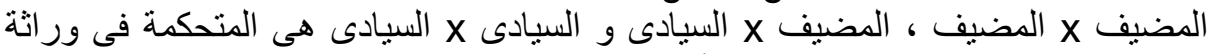

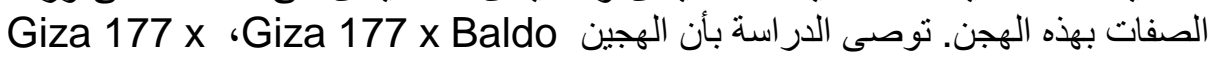
'Large stigma SKC23819-192 x Baldo و Giza 178 x Large stigma بر امج تربية الأرز لتحسن صفات المحصول ومكوناته والمقاوة لمرض اللفحة. 